\title{
Regioselective Difunctionalization of 2,6-Difluorophenols Triggered by Sigmatropic Dearomatization
}

\section{$\operatorname{AUTHOR}(\mathrm{S}):$}

Okamoto, Koichi; Nogi, Keisuke; Yorimitsu, Hideki

\section{CITATION:}

Okamoto, Koichi ...[et al]. Regioselective Difunctionalization of 2,6-Difluorophenols Triggered by Sigmatropic Dearomatization. Organic Letters 2020, 22(14): 5540-5544

\section{ISSUE DATE:}

2020-07-17

URL:

http://hdl.handle.net/2433/255853

\section{RIGHT:}

This document is the Accepted Manuscript version of a Published Work that appeared in final form in Organic Letters, copyright $\odot$ American Chemical Society after peer review and technical editing by the publisher. To access the final edited and published work see https://doi.org/10.1021/acs.orglett.0c01904.; The full-text file will be made open to the public on 7 July 2021 in accordance with publisher's 'Terms and Conditions for Self-Archiving'., この論文は出版社版て ありません。引用の際には出版社版をじ確認ご利用ください。;This is not the published version. Please cite only the published version. 


\title{
Regioselective Difunctionalization of 2,6-Difluorophenols Trig- gered by Sigmatropic Dearomatization
}

\author{
Koichi Okamoto, Keisuke Nogi, and Hideki Yorimitsu* \\ Department of Chemistry, Graduate School of Science, Kyoto University, Sakyo-ku, Kyoto, 606-8502, Japan \\ Supporting Information Placeholder
}

(c)

\begin{abstract}
Regioselective difunctionalization of 2,6-difluorophenols with aryl sulfoxides and nucleophiles has been accomplished. The reaction is composed of (1) Pummerer-based [3,3] sigmatropic dearomatization to generate 2,4-cyclohexadienone, (2) Michael addition of a nucleophile, and (3) liberation of HF for rearomatization. Besides the [3,3] rearrangement, [2,3] sigmatropic rearrangement from sulfonium ylide generated from alkyl sulfoxide promotes the dearomatization resulting in installation of $\alpha$-sulfanylalkyl group.
\end{abstract}

Organofluorine compounds have occupied a privileged position in the field of pharmaceuticals, agrochemicals, and optoelectronics. ${ }^{1}$ Significant effort has been devoted for the synthesis of a wide range of organofluorine compounds. ${ }^{2,3,4}$ Selective $\mathrm{C}-$ $\mathrm{F}$ transformations are among the most important methods because readily available polyfluorinated arenes can be employed as starting materials. ${ }^{3}$ Besides classical $\mathrm{S}_{\mathrm{N}} \mathrm{Ar}$ reactions, ${ }^{4}$ recent progress in the cross-coupling arena has allowed transitionmetal-mediated and catalyzed C-F functionalization.

As a totally different approach, we are interested in sigmatropic dearomatization/defluorination sequences toward selective $\mathrm{C}-\mathrm{F}$ bond transformation of polyfluorophenols. ${ }^{5}$ The reaction is initiated by interrupted Pummerer reaction of alkenyl ${ }^{5 a}$ or aryl ${ }^{5 b}$ sulfoxides with polyfluorophenols with the aid of trifluoroacetic anhydride (TFAA) (Scheme 1a). ${ }^{6}$ Subsequent $[3,3]$ sigmatropic rearrangement would furnish dearomatized intermediate $\mathbf{A},{ }^{7}$ which is eventually involved in reductive defluorination with $\mathrm{Zn}$ powder to accomplish C-F transformations of fluorophenols (Scheme 1a, top). We envisioned that $\mathbf{A}$ can be a competent intermediate for Michael addition owing to the fairly reactive 2,4-cyclohexadienone skeleton of $\mathbf{A}$. In analogy with the reaction of ortho-quinone monoacetals, ${ }^{8}$ a nucleophile would add to the 3 position of $\mathbf{A}$, which would be followed by rearomatization with the loss of HF (Scheme 1a, bottom). This transformation can be regarded as an unusual 2,5-difunctionalization of 2,6-difluorophenol. Similar difunctionalization via a sequence of sigmatropic dearomatization/Michael addition was reported by Peng by using aryliodanes (Scheme 1b). ${ }^{9,10}$ However, the Michael addition products still have dearomatized skeleton owing to the absence of leaving groups. On the other hand, in our system, fluorine serves as a leaving group for rearomatization, while its poor leaving ability inhibits too fast decomposition of $\mathbf{A}$. We herein report regioselective difunctionalization of 2,6-difluorophenols based on sigmatropic dearomatization. With aryl sulfoxides and nucleophiles including amines and thiols, multifunctionalized phenols can be synthesized.

Scheme 1. Sigmatropic dearomatization toward difunctionalization of aromatic compounds

a) 2,5-difunctionalization of fluorophenols<smiles>C[Sb](=O)c1ccccc1</smiles><smiles>COc1ccccc1C1(F)C=CC=C(F)C1=O</smiles>
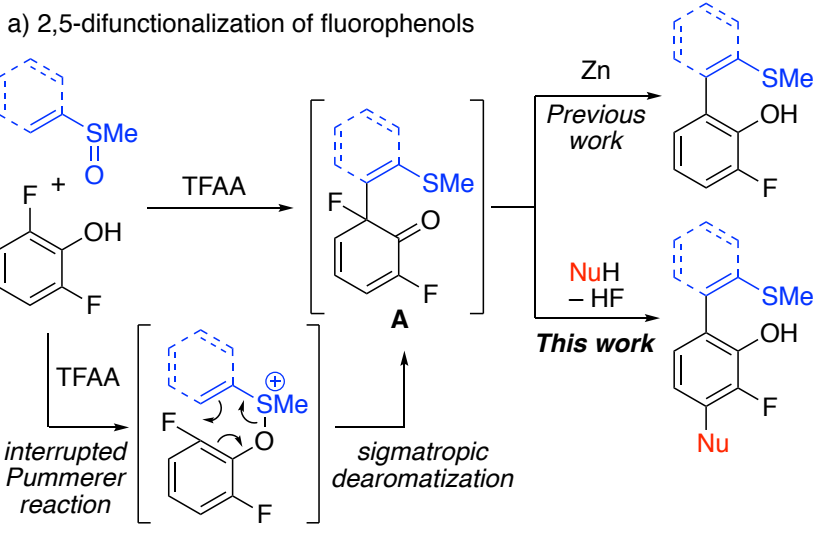

b) dearomative 2,5-difunctionalization of aryliodanes

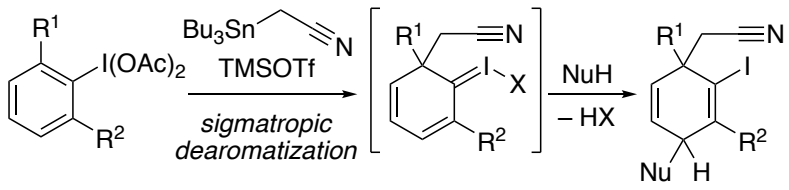


Based on the hypothesis, dearomatized intermediate $\mathbf{A}$ was generated from 2,6-difluorophenol and 2-benzothienyl sulfoxide 1a under conditions similar to those for our previous $\mathrm{C}-\mathrm{F}$ arylation $^{5 \mathrm{~b}}$ except for the absence of $\mathrm{Zn}$ powder. ${ }^{11}$ Subsequently, piperidine ( 6.0 equiv) was added, and the solution was allowed to warm to room temperature and stirred for $1 \mathrm{~h}$. Gratifyingly, the desired difunctionalized fluorophenol 2a was obtained in $71 \%$ yield (Scheme 2). The excess amount of piperidine was employed not only as a nucleophile but also as a base to neutralize $\mathrm{CF}_{3} \mathrm{CO}_{2} \mathrm{H}$ generated via the interrupted Pummerer reaction. Instead of piperidine, other secondary amines, pyrrolidine and diethylamine, furnished the corresponding difunctionalization products $\mathbf{2 b}$ and $\mathbf{2 d}$ in $74 \%$ and $68 \%$ yields, respectively. The present method is applicable to gram-scale synthesis: for example, $1.5 \mathrm{~g}$ (67\% yield) of $\mathbf{2 b}$ was obtained from $6.0 \mathrm{mmol}$ of 1a. Benzothienyl $p$-tolyl sulfoxide $\mathbf{1 b}$ also underwent the reaction to afford $2 \mathrm{c}$ in $76 \%$ yield. Primary amines were also suitable for the reaction to afford $\mathbf{2 e}$ and $\mathbf{2 f}$, albeit the use of bulky $t$-butylamine provided $\mathbf{2 g}$ in $37 \%$ yield. Unfortunately, aniline was not a competent nucleophile probably because of the lower nucleophilicity. The reactions with 4-chloro-2,6-difluorophenol and with 2,6-difluoro-4-iodophenol afforded the corresponding fluorophenols $\mathbf{2} \mathbf{i}$ and $\mathbf{2} \mathbf{j}$ in moderate yields, respectively, in spite of the steric hindrance at the 5-position. With respect to aryl sulfoxides, 2-benzofuryl and 2-indolyl sulfoxides 1c and 1d smoothly underwent the reaction to afford $\mathbf{2 k}$ and $\mathbf{2} \mathbf{l}$, respectively, under slightly modified conditions.

\section{Scheme 2. Substrate scope}
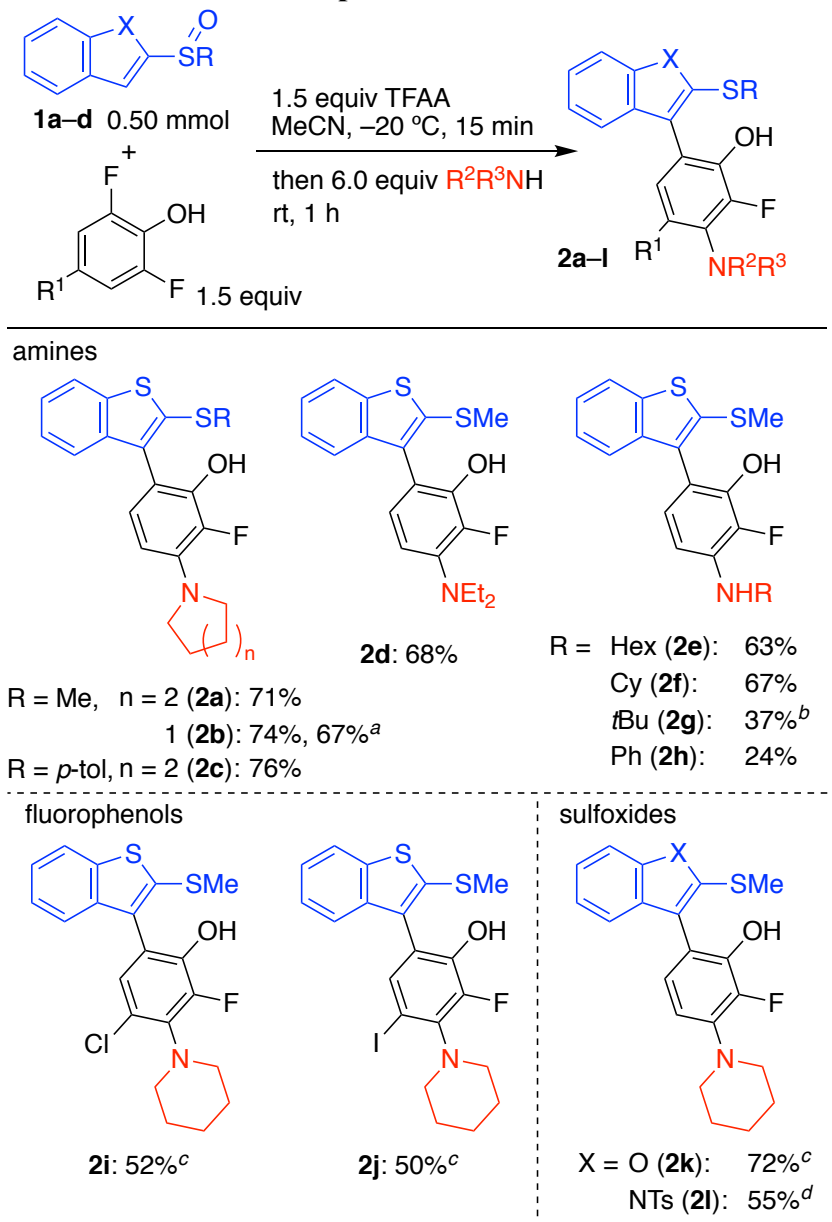

${ }^{a} 6.0 \mathrm{mmol}$ scale. ${ }^{b}$ Stirred for $3 \mathrm{~h}$ in the second step. ${ }^{c}$ The first step was conducted at $-40{ }^{\circ} \mathrm{C}$. ${ }^{d} 2.0$ equiv of TFAA were used.
When methyl 3-thienyl sulfoxide was employed, unexpected fluorophenol 3a was formed in 14\% NMR yield instead of fluorophenol $\mathbf{2 m}$ (Scheme 3). It is speculated that the stronger aromaticity of thiophene ring than that of benzothiophene ring inhibited [3,3] sigmatropic rearrangement of sulfonium B. Instead, deprotonation at the methyl group of $\mathbf{B}$ would form sulfonium ylide $\mathbf{C}$, which would then undergo [2,3] sigmatropic rearrangement ${ }^{12}$ to furnish dearomatized intermediate $\mathbf{A}^{\prime}$. Finally, Michael addition of piperidine to $\mathbf{A}^{\prime}$ followed by elimination of HF would afford $\mathbf{3 a}$.

Scheme 3. Unexpected $[2,3]$ sigmatropic rearrangement
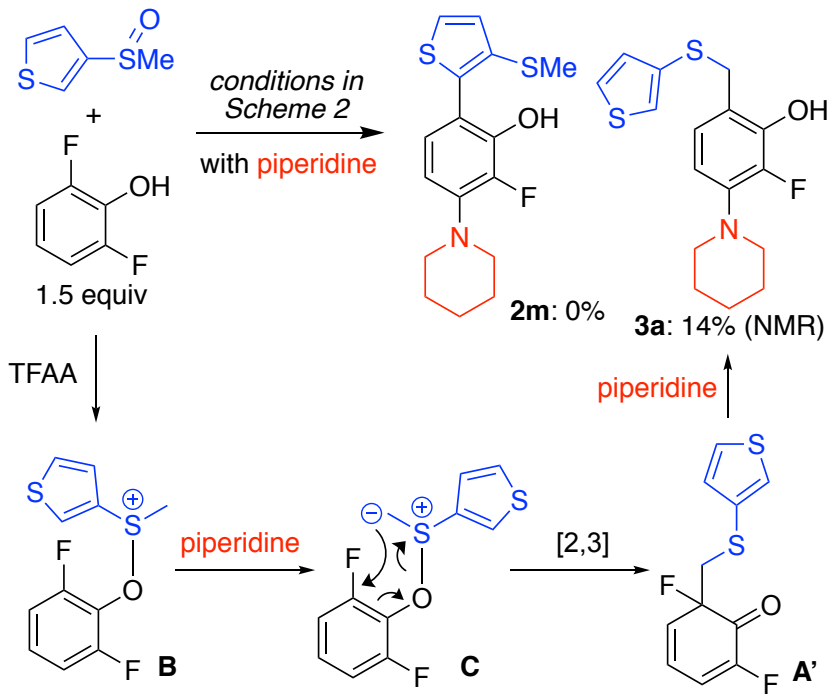

This finding inspired us to further investigate this tandem $\mathrm{C}-$ $\mathrm{F}$ alkylation/C-H amination. By using triethylamine for the deprotonation of $\mathbf{B}$, the $\mathrm{C}-\mathrm{F}$ alkylation uneventfully proceeded to furnish $\mathbf{3 a}$ in $70 \%$ yield. (Scheme 4 ). The reaction with DMSO smoothly proceeded to afford $3 \mathbf{b}$ in $68 \%$ yield. The structure of $\mathbf{3 b}$ was unambiguously confirmed by X-ray crystal structure analysis. ${ }^{13}$ Methyl phenyl sulfoxide exclusively provided $3 \mathbf{c}$ generated via [2,3] sigmatropic rearrangement in 58\% yield. Trisubstituted carbon center could also be constructed with dibutyl sulfoxide, and the corresponding fluorophenol 3d was obtained in $56 \%$ yield.

\section{Scheme 4. Utilization of $[2,3]$ sigmatropic rearrangement}
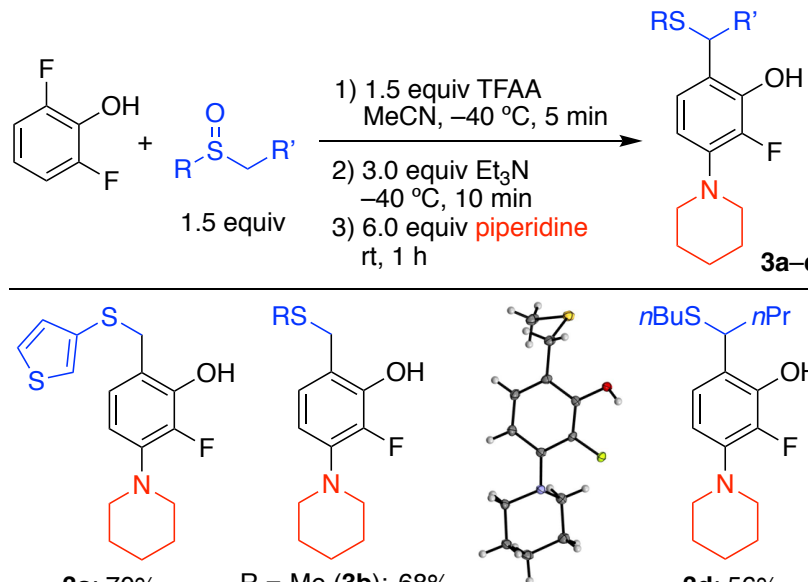

3a: $70 \%$

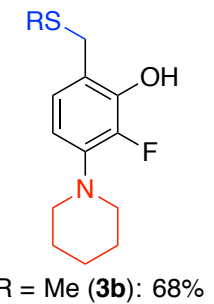

$\mathrm{Ph}(3 \mathrm{c}): 58 \%$

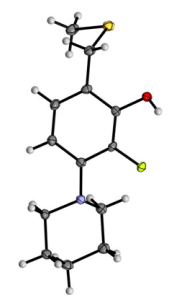

$3 b$

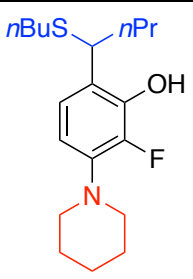

3d: $56 \%$ 
We next explored the introduction of a different class of nucleophiles. In consequence, it was found that thiols reacted smoothly in the presence of triethylamine (Scheme 5). The reaction of 2,6-difluorophenol with sulfoxide 1a followed by treatment with $p$-toluenethiol afforded the corresponding 2,5difunctionalized product $4 \mathbf{a}$ in $67 \%$ yield along with a tiny amount of 4-sulfanylated product $\mathbf{4} \mathbf{a}^{\prime}$ presumably formed via $\mathrm{S}_{\mathrm{N}} 2$ ' reaction of dearomatized intermediate with the thiol. The employment of 4-chloro-2,6-difluorophenol uneventfully provided $\mathbf{4 b}$ in $57 \%$ yield. Alkanethiol was also applicable to the reaction to yield $\mathbf{4 c}$. The intermediate generated via $[2,3]$ rearrangement could be involved in the reaction with $p$-toluenethiol to provide 5 in $59 \%$ yield.

\section{Scheme 5. Addition of thiols}
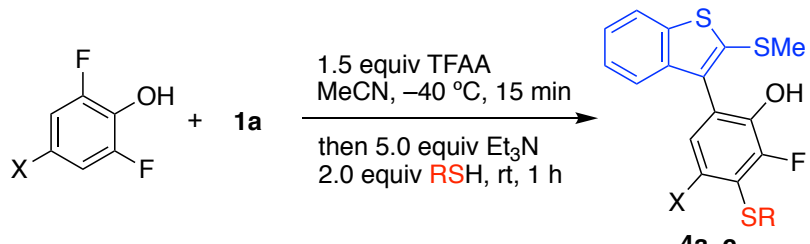

$4 a-c$
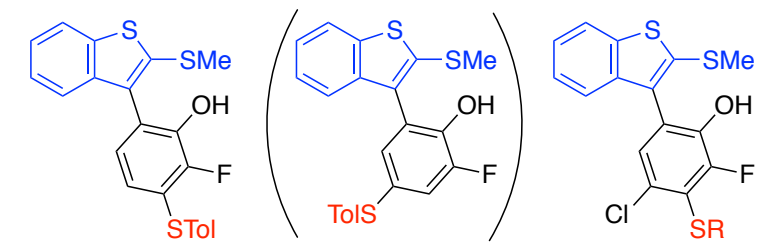

4a: $70 \%^{a}$

$4 a^{\prime}$

$$
\mathrm{R}=\mathrm{Tol}(\mathbf{4 b}): \quad 57 \%
$$

$n \mathrm{C}_{12} \mathrm{H}_{25}(4 \mathrm{c}): 47 \%$

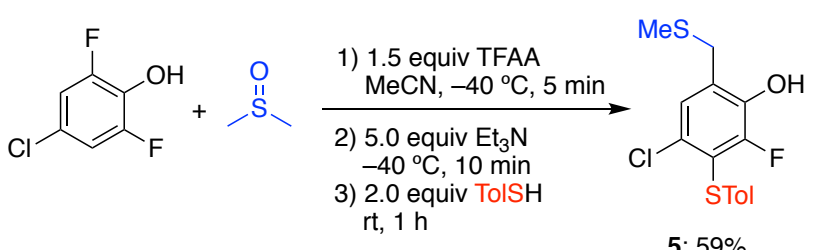

${ }^{a}$ Containing a tiny amount of 4-sulfanylated phenol $\mathbf{4} \mathbf{a}^{\prime}(>20 / 1)$.

Scheme 6. Competition experiment
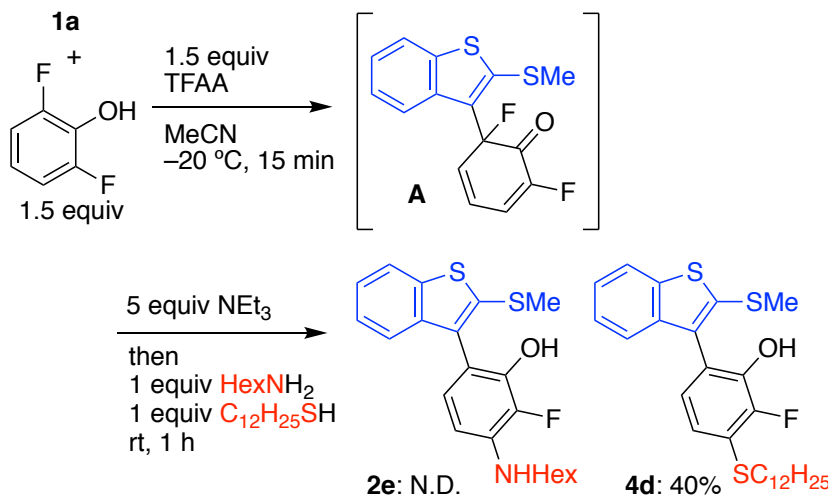

(NMR)

To investigate relative reactivities of nucleophiles, we conducted a competition experiment by using hexylamine and dodecanethiol. Although sulfanylated product $\mathbf{4 d}$ was formed in $40 \%$ NMR yield, the corresponding aminated product $2 \mathbf{e}$ was not detected after the reaction, which indicates the higher reactivity of the thiol rather than the amine toward dearomatized intermediate $\mathbf{A}$ (Scheme 6).

To check the indispensability of the ortho-fluoro substituents, we tried the reaction with 2-fluorophenol. The reaction with 1a and piperidine under the conditions in Scheme 2 provided a mixture of the desired difunctionalized product $\mathbf{2 n}$ and biaryl 20 in a ratio of 1.4:1 (Scheme 7a). The latter is generated through $\mathrm{C}-\mathrm{C}$ bond formation at the expense of the $\mathrm{C} 6-\mathrm{H}$ bond of 2-fluorophenol. Similar non-regioselective $\mathrm{C}-\mathrm{C}$ bond formation was observed in our previous work. ${ }^{5}$ In place of 2,6difluorophenol, the use of 2,6-dichlorophenol failed to deliver difunctionalized product $\mathbf{2 p}$ and gave a complex product mixture (Scheme $7 b$ ). This result suggests that decomposition of dearomatized intermediate A-Cl would be too fast to survive until the addition of piperidine. This result highlights the essential role of fluorine, i.e. balancing the stability of the cyclohexadienone intermediate and the facileness of rearomatization. We also tried the reaction with 2,6-dimethylphenol. Although $\mathrm{C}-\mathrm{C}$ bond formation with $1 \mathrm{a}$ via dearomatization proceeded, subsequent Michael addition of piperidine did not occur resulting in the formation of cyclohexadienone $2 r$ in $59 \%$ NMR yield (Scheme 7c). The electron-donating nature and/or the larger size of methyl group compared to fluoro group might hamper the Michael addition process.

Scheme 7. Attempted difunctionalizations of phenols other than 2,6-difluorinated ones
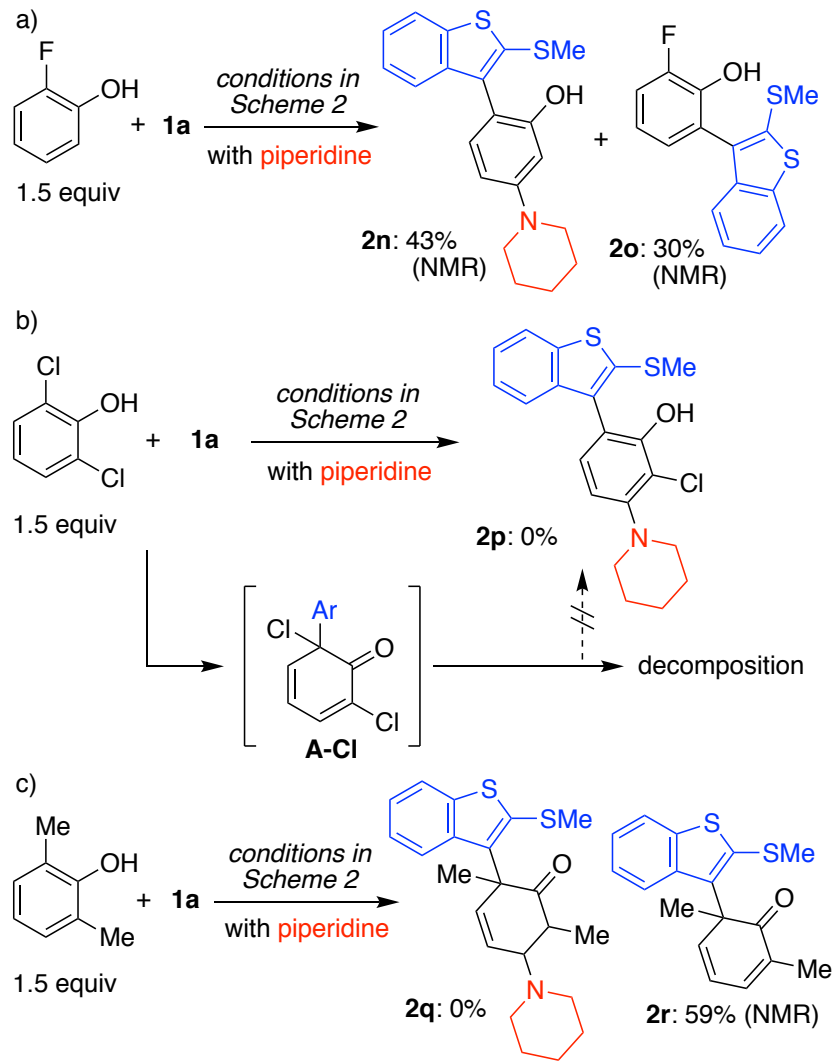

Finally, we conducted the reaction with 2,3,5,6-tetrafluorophenol. Under the optimized conditions, tetrafluorophenol successively reacted with $\mathbf{1 a}$ and piperidine. As a result, cyclohexadiene 6 was obtained in 39\% yield (Scheme 8a). The sigmatropic dearomatization and subsequent Michael addition would afford intermediate $\mathbf{D}$ and the following elimination of $\mathrm{HF}$ at the expense of C5-F bond would form $\mathbf{6}$. Notably, besides $\mathbf{6}$, 
another cyclohexadienone 7 was also detected in the product mixture. We assumed that 7 would be generated via 1,6-addition of the remaining piperidine and subsequent elimination of HF. Encouraged by these findings, we attempted two-step 2,3,5-trifunctionalization of tetrafluorophenol (Scheme 8b). First, tetrafluorophenol was successively treated with $\mathbf{1 a}$ and TFAA, piperidine, and $p$-toluenethiol to furnish cyclohexadienone 8. The reduction of $\mathbf{8}$ with $\mathrm{Zn}$ powder afforded the desired trifunctionalized product 9 in $67 \%$ overall yield. ${ }^{14}$ Remarkably, 9 has a benzene ring substituted by six different elements.

\section{Scheme 8. 2,3,5-Trifunctionalization of tetrafluorophenol}

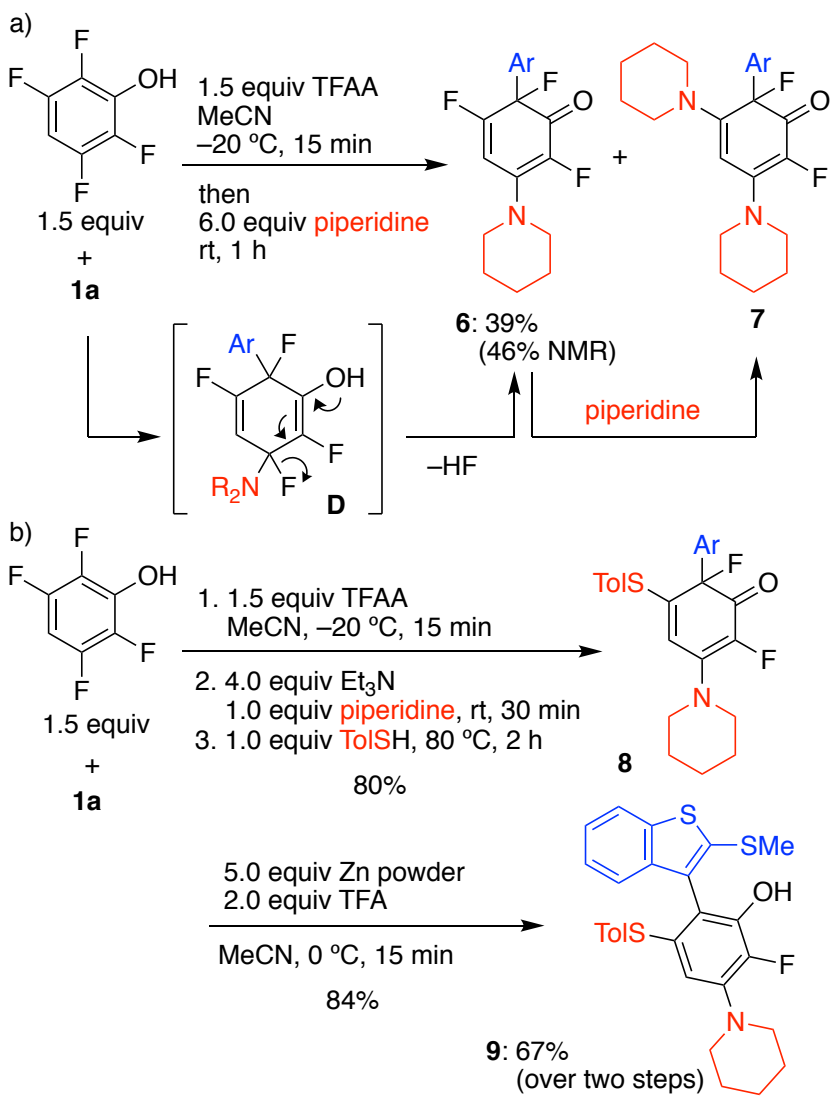

In conclusion, we have achieved unusual 2,5-difunctionalization of 2,6-difluorophenols in a metal-free manner, based on sigmatropic dearomatization and subsequent Michael addition of nucleophiles. The reaction is realized by fluorine as a poor leaving group, which hinders fast decomposition of the dearomatized intermediate, but smoothly departs on rearomatization.

\section{ASSOCIATED CONTENT}

\section{Supporting Information}

Experimental procedures, X-ray crystallographic analysis, and spectral data (PDF)

\section{AUTHOR INFORMATION}

\section{Corresponding Author}

*yori@kuchem.kyoto-u.ac.jp

\section{Notes}

The authors declare no competing financial interest.

\section{ACKNOWLEDGMENT}

This work was supported by JSPS KAKENHI Grant Numbers JP18H04254, JP18H04409, JP19H00895, and JP18K14212 as well as JST CREST Grant Number JPMJCR19R4. Mr. Akira Yoshida is acknowledged for performing the gram-scale synthesis. H.Y. thanks The Mitsubishi Foundation for financial support.

\section{REFERENCES}

(1) (a) Hiyama, T. Organofluorine Compounds. Chemistry and Applications; Springer: Berlin, 2000. (b) Chambers, R. D. Fluorine in Organic Chemistry; Blackwell: Oxford, 2004. (c) Kirsch, P. Modern Fluoroorganic Chemistry. Synthesis, Reactivity, Applications; WileyVCH: Weinheim, 2013. (d) Müller, K.; Faeh, C.; Diederich, F. Fluorine in Pharmaceuticals: Looking Beyond Intuition. Science 2007, 317, 1881. (e) Kirk, K. L. Fluorination in Medicinal Chemistry: Methods, Strategies, and Recent Developments. Org. Process Res. Dev. 2008, 12, 305. (f) Purser, S.; Moore, P. R.; Swallow, S.; Gouverneur, V. Fluorine in medicinal chemistry. Chem. Soc. Rev. 2008, 37, 320. (g) Hagmann, W. K. The Many Roles for Fluorine in Medicinal Chemistry. J. Med. Chem. 2008, 51, 4359. (h) Wang, J.; Sánchez-Roselló, M.; Aceña, J. L.; del Pozo, C.; Sorochinsky, A. E.; Fustero, S.; Soloshonok, V. A.; Liu, H. Fluorine in Pharmaceutical Industry: Fluorine-Containing Drugs Introduced to the Market in the Last Decade (2001-2011). Chem. Rev. 2014, 114, 2432.

(2) Recent reviews on the synthesis of fluorinated molecules via $\mathrm{C}-$ F bond formation: (a) Ma, J.-A.; Li, S. Catalytic fluorination of unactivated $\mathrm{C}\left(\mathrm{sp}^{3}\right)-\mathrm{H}$ bonds. Org. Chem. Front. 2014, 1, 712. (b) Campbell, M. G.; Ritter, T. Modern Carbon-Fluorine Bond Forming Reactions for Aryl Fluoride Synthesis. Chem. Rev. 2015, 115, 612. (c) Champagne, P. A.; Desroches, J.; Hamel, J.-D.; Vandamme, M.; Paquin, J.F. Monofluorination of Organic Compounds: 10 Years of Innovation. Chem. Rev. 2015, 115, 9073. (d) Sather, A. C.; Buchwald, S. L. The Evolution of $\mathrm{Pd}^{0} / \mathrm{Pd}^{\mathrm{II}}$-Catalyzed Aromatic Fluorination. Acc. Chem. Res. 2016, 49, 2146. (e) Petrone, D. A.; Ye, J.; Lautens, M. Modern Transition-Metal-Catalyzed Carbon-Halogen Bond Formation. Chem. Rev. 2016, 116, 8003. (f) Shibata, N. Development of Shelf-Stable Reagents for Fluoro-Functionalization Reactions. Bull. Chem. Soc. Jpn. 2016, 89, 1307.

(3) Selected reviews on the synthesis of fluorinated molecules via metal-mediated or -catalyzed C-F cleavage: (a) Amii, H.; Uneyama, K. $\mathrm{C}-\mathrm{F}$ Bond Activation in Organic Synthesis. Chem. Rev. 2009, 109, 2119. (b) Kuehnel, M. F.; Lentz, D.; Braun, T. Synthesis of Fluorinated Building Blocks by Transition-Metal-Mediated Hydrodefluorination Reactions. Angew. Chem. Int. Ed. 2013, 52, 3328. (c) Ahrens, T.; Kohlmann, J.; Ahrens, M.; Braun, T. Functionalization of Fluorinated Molecules by Transition-Metal-Mediated C-F Bond Activation To Access Fluorinated Building Blocks. Chem. Rev. 2015, 115, 931. (d) Shen, Q.; Huang, Y.-G.; Liu, C.; Xiao, J.-C.; Chen, Q.-Y.; Guo, Y. Review of recent advances in $\mathrm{C}-\mathrm{F}$ bond activation of aliphatic fluorides. J. Fluorine Chem. 2015, 179, 14. (e) Chen, W.; Bakewell, C.; Crimmin, M. R. Functionalisation of Carbon-Fluorine Bonds with Main Group Reagents. Synthesis 2017, 49, 810.

(4) Liu, C.; Zhang, B. Facile Access to Fluoroaromatic Molecules by Transition-Metal-Free C-F Bond Cleavage of Polyfluoroarenes: An Efficient, Green, and Sustainable Protocol. Chem. Rec. 2016, 16, 667.

(5) (a) Okamoto, K.; Hori, M.; Yanagi, T.; Murakami, K.; Nogi, K.; Yorimitsu, H. Sigmatropic Dearomatization/Defluorination Strategy for $\mathrm{C}-\mathrm{F}$ Transformation: Synthesis of Fluorinated Benzofurans from Polyfluorophenols. Angew. Chem. Int. Ed. 2018, 57, 14230. (b) Okamoto, K.; Nogi, K.; Shimokawa, J.; Yorimitsu, H. C-F Arylation of Polyfluorophenols by Means of Sigmatropic Dearomatization/Defluorination Sequence. Chem. Eur. J. 2020, 26, 5615.

(6) Selected transformations of aryl and alkenyl sulfoxides with phenols utilizing interrupted Pummerer reaction: (a) Kobatake, T.; Fujino, D.; Yoshida, S.; Yorimitsu, H.; Oshima, K. Synthesis of 3-Trifluoromethylbenzo $[b]$ furans from Phenols via Direct Ortho Functionalization by Extended Pummerer Reaction. J. Am. Chem. Soc. 2010, 132, 11838. (b) Yanagi, T.; Otsuka, S.; Kasuga, Y.; Fujimoto, K.; Murakami, K.; Nogi, K.; Yorimitsu, H.; Osuka, A. Metal-Free Approach to Biaryls 
from Phenols and Aryl Sulfoxides by Temporarily Sulfur-Tethered Regioselective C-H/C-H Coupling. J. Am. Chem. Soc. 2016, 138, 14582. (c) Shrives, H. J.; Fernández-Salas, J. A.; Hedtke, C.; Pulis, A. P.; Procter, D. J. Regioselective synthesis of C3 alkylated and arylated benzothiophenes. Nat. Commun. 2017, 8, 14801. (d) Chen, D.; Feng, Q.; Yang, Y.; Cai, X.-M.; Wang, F.; Huang, S. Metal-free O-H/C-H difunctionalization of phenols by $o$-hydroxyarylsulfonium salts in water. Chem. Sci. 2017, 8, 1601. (e) He, Z.; Shrives, H. J.; FernándezSalas, J. A.; Abengózar, A.; Neufeld, J.; Yang, K.; Pulis, A. P.; Procter, D. J. Synthesis of C2 Substituted Benzothiophenes via an Interrupted Pummerer/[3,3]-Sigmatropic/1,2-Migration Cascade of Benzothiophene S-Oxides. Angew. Chem. Int. Ed. 2018, 57, 5759. (f) Yang, K.; Pulis, A. P.; Perry, G. J. P.; Procter, D. J. Transition-Metal-Free Synthesis of C3-Arylated Benzofurans from Benzothiophenes and Phenols. Org. Lett. 2018, 20, 7498. (g) Hori, M.; Yanagi, T.; Murakami, K.; Nogi, K.; Yorimitsu, H. Annulative Synthesis of Benzofurans from General Alkenyl Sulfoxides and Phenols via Pummerer/Sigmatropic Cascade. Bull. Chem. Soc. Jpn. 2019, 92, 302. (h) Yanagi, T.; Nogi, K.; Yorimitsu, H. Sulfoxide-Directed Iterative Assembly into Oligoarenes. Synlett 2020, 31, 153.

(7) Reviews on transformations utilizing interrupted Pummerer reaction/ $[3,3]$ sigmatropic rearrangement sequences: (a) Smith, L. H. S.; Coote, S. C.; Sneddon, H. F.; Procter, D. J. Beyond the Pummerer Reaction: Recent Developments in Thionium Ion Chemistry. Angew. Chem. Int. Ed. 2010, 49, 5832. (b) Shafir, A. The emergence of sulfoxide and iodonio-based redox arylation as a synthetic tool. Tetrahedron Lett. 2016, 57, 2673. (c) Pulis, A. P.; Procter, D. J. C-H Coupling Reactions Directed by Sulfoxides: Teaching an Old Functional Group New Tricks. Angew. Chem. Int. Ed. 2016, 55, 9842. (d) Yorimitsu, H. Cascades of Interrupted Pummerer Reaction - Sigmatropic Rearrangement. Chem. Rec. 2017, 17, 1156. (e) Yanagi, T.; Nogi, K.; Yorimitsu, $\mathrm{H}$. Recent development of ortho-C-H functionalization of aryl sulfoxides through $[3,3]$ sigmatropic rearrangement. Tetrahedron Lett. 2018, 59, 2951. (f) Kaiser, D.; Klose, I.; Oost, R.; Neuhaus, J.; Maulide, N. Bond-Forming and -Breaking Reactions at Sulfur (IV): Sulfoxides, Sulfonium Salts, Sulfur Ylides, and Sulfinate Salts. Chem. Rev. 2019, 119, 8701. (g) He, Z.; Pulis, A. P.; Perry, G. J. P.; Procter, D. J. Pummerer chemistry of benzothiophene S-oxides: Metal-free alkylation and arylation of benzothiophenes. Phosphorus Sulfur Silicon Relat. Elem. 2019, 194, 669. (h) Zhang, L.; Hu, M.; Peng, B. [3,3]- and [5,5]Sigmatropic Rearrangements of Aryl Sulfoxides Using An 'Assembly/Deprotonation' Technology. Synlett 2019, 30, 2203.

(8) (a) Hsieh, M,-F.; Rao, P. D.; Liao, C.-C. Diels-Alder and Michael addition reactions of indoles with masked o-benzoquinones: synthesis of highly functionalized hydrocarbazoles and 3-arylindoles.
Chem. Commun. 1999, 1441. (b) Chittimalla, S. K.; Bandi, C.; Putturu, S.; Kuppusamy, R.; Boellaard, K. C.; Tan, D. C. A.; Lum, D. M. J. Access to 3-Arylindoles through a Tandem One-Pot Protocol Involving Dearomatization, a Regioselective Michael Addition Reaction, and Rearomatization. Eur. J. Org. Chem. 2014, 12, 2565. (c) Chittimalla, S. K.; Kuppusamy, R.; Bandi, C. A Detour Route for meta Functionalization of Phenols. Synlett 2014, 25, 1991. (d) Parumala, S. K. R.; Surasani, S. R.; Peddinti, R. K. S-Arylation of thiols with masked o-benzoquinones: synthesis of alkyl aryl/diaryl sulfides. New J. Chem. 2014, 38, 5268. (e) Chittimalla, S. K.; Kuppusamy, R.; Akavaram, N. PalladiumCatalyzed Regioselective Synthesis of Oxygenated Biphenyls. Synlett 2015, 26, 613. (f) Chittimalla, S. K.; Bandi, C. Tandem Oxidative Phenol Dearomatization/Formal [3+2] Annulation Protocol En Route to Highly Functionalized Benzothiophenes. Synlett 2017, 28, 1051. (g) Chittimalla, S. K.; Bandi, C.; Gadi, V. K.; Gunturu, S. R. Facile Synthesis of 2-Arylindoles through Plancher-Type Rearrangement of 3-Alkyl-3-Arylindolenines. Synlett 2017, 28, 1994. (h) Chittimalla, S. K.; Nakka, S.; Koodalingam, M.; Bandi, C. N-Arylation of Heterocycles by a Tandem Aza-Michael Addition Reaction and Aromatization Sequence. Synlett 2018, 29, 57.

(9) Zhao, W.; Huang, X.; Zhan, Y.; Zhang, Q.; Li, D.; Zhang, Y.; Kong, L.; Peng, B. Dearomative Dual Functionalization of Aryl Iodanes. Angew. Chem. Int. Ed. 2019, 58, 17210.

(10) A related report from Peng group: Huang, X.; Zhang, Y.; Liang, W.; Zhang, Q.; Zhan, Y.; Kong, L.; Peng, B. Dearomatization of aryl sulfoxides: a switch between mono- and dual-difluoroalkylation. Chem. Sci. 2020, 11, 3048.

(11) Generation of intermediate A was confirmed by ${ }^{1} \mathrm{H}$ NMR. For details, see Figure S1 in the Supporting Information.

(12) $[2,3]$ sigmatropic rearrangement from similar sulfonium ylides: (a) Sato, K.; Inoue, S.; Ozawa, K. Selective ortho-methylthiomethylation of phenols with dimethyl sulphoxide and thionyl chloride. J. Chem. Soc. Perkin Trans. 1 1984, 2715. (b) Sato, K.; Inoue, S.; Ozawa, K.; Kobayashi, T.; Ota, T. Selective ortho-alkylation of phenols with sulphoxides via $[2,3]$ sigmatropic rearrangement: synthesis of coumarins. J. Chem. Soc. Perkin Trans. 1 1987, 1753. (c) Sato, K.; Inoue, S.; Miyamoto, O.; Ikeda, H.; Ota, T. Ortho-Selective Alkylation of Phenols with Symmetric Sulfides and Sulfuryl Chloride. Bull. Chem. Soc. Jpn. 1987, 60, 4184. (d) Hu, G.; Xu, J.; Li, P. Sulfur mediated propargylic C-H alkylation of alkynes. Org. Chem. Front. 2018, 5, 2167.

(13) For detailed information, see the Supporting Information.

(14) Instead of the Zn-promoted reduction, we attempted 1,2-aryl migration of $\mathbf{8}$ (cf. Ref. $5 \mathrm{~b}$ ). However, treatment of $\mathbf{8}$ with 2 equiv of $\mathrm{BF}_{3} \cdot \mathrm{Et}_{2} \mathrm{O}$ did not facilitate the 1,2-aryl migration and $74 \%$ of 8 was recovered after the reaction. 